\title{
Mehr Ärzte, aber schlechte Verteilung
}

Im internationalen Vergleich gibt es in Deutschland überdurchschnittlich viele praktizierende Ärzte. Das ist ein zentrales Ergebnis aus dem aktuellen Ärzteatlas 2016 des Wissenschaftlichen Instituts der Allgemeinen Ortskrankenkasse (AOK), kurz WIdO. In der ambulanten vertragsärztlichen Versorgung gibt es demnach keinen Mangel, sondern oftmals sogar eine Überversorgung.Mit 4,1 Ärzten pro 1000 Einwohner liegt Deutschland der Erhebung zufolge knapp ein Viertel über dem internationalen Durchschnitt (3,3 Ärzte). Zwischen 1991 und 2015 ist die Arztdichte um 50 Prozent gestiegen.

Laut Ärzteatlas wird in allen 34 erfassten Fachrichtungen das Plansoll bundesweit um fast ein Drittel übertroffen. Als Grundlage dienen die Regeln der aktuellen Bedarfsplanung für die ambulante vertragsärztliche Versorgung.

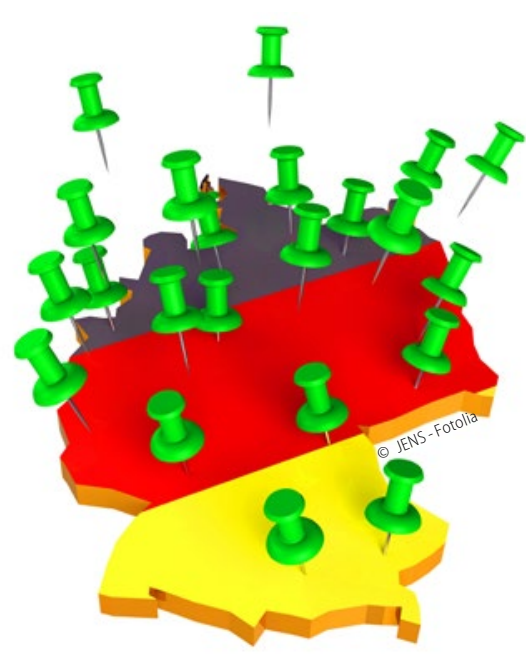

Der stellvertretende WIdO-Geschäftsführer, Helmut Schröder, wies bei der Vorstellung der Ergebnisse aber auf regionale Unterschiede hin: „Einer Unterversorgung oder drohenden Unterversorgung in einigen Landstrichen steht eine deutliche Überversorgung insbesondere in Ballungsgebieten und für Ärzte attraktiven Regionen gegenüber.“ Die Überversorgung binde Ressourcen, die an anderer Stelle fehlten, betonte Schröder.

Die Kassenärztliche Bundesvereinigung (KBV) kritisierte die Erhebung und hielt mit eigenen Zahlen dagegen. Demnach habe sich die Gesamtzahl der Ärzte und Psychotherapeuten an der vertragsärztlichen Versorgung im vergangenen Jahr zwar um 1,4 Prozent erhöht, aber dieser Anstieg spiegele sich lediglich geringfügig in der Anzahl der geleisteten Arztstunden wider (plus 0,2 Prozent). Die KBV begründete die Unterschiede damit, dass der AOK-Ärzteatlas aktuelle Entwicklungen wie Anstellung und Teilzeit nicht berücksichtige.

\section{Online-Datenbank}

\section{Auskunft über Zahlungen der Pharmafirmen an Mediziner}

In einer bundesweiten Datenbank haben Spiegel Online und das Recherchezentrum Correctiv zusammengetragen, welcher Arzt welche Gelder im vergangenen Jahr von Pharmafirmen erhalten hat. Die Daten basieren auf freiwilligen Angaben im Rahmen des Transparenzkodexes des Verbandes der forschenden Pharma-Unternehmen (vfa). Insgesamt flossen 575 Millionen Euro an 71.000 Mediziner und Fachkreisangehörige sowie 6200 medizinische Einrichtungen. Davon stimmte ein Drittel der
Veröffentlichung zu. Über die Suchkriterien Name, Ort oder Postleitzahl können die 20.000 Ärzte, die der Veröffentlichung zustimmten, in der Online-Datenbank eingesehen werden. Aus diesen Angaben geht hervor, dass die Pharmaindustrie durchschnittlich 1646 Euro an Ärzte zahlte. Die vfa-Hauptgeschäftsführerin Birgit Fischer lobte die Transparenzoffensive: „Auf Basis dieser Zahlen kann die Öffentlichkeit nachvollziehen, wie Ärzte und Pharmaunternehmen zusammenarbeiten.“

\section{DAK-Gesundheit}

\section{Kasse stellt Zahlungen für wechselwillige Mitglieder in Aussicht}

Der Krankenkasse DAK-Gesundheit droht neuer Ärger: Nach einem Bericht des Nachrichtenmagazins „Der Spiegel“ soll die Kasse ihren Versicherten eine Prämie für den Verbleib in der DAK-Gesundheit gezahlt haben. Damit könnte die Kasse gegen die Wettbewerbsgrundsätze verstoßen haben. Nach Spiegel-Informationen wurde den Versicherten eine Prämie versprochen, wenn sie ihre Kündigung der Mitgliedschaft zurücknehmen oder die Teilnahme an einer Marktforschung signalisieren. Diese Praktiken hatten mehrere Kassen gegenüber dem Bundesversicherungsamt angezeigt. Gegenüber der „Ärzte Zeitung“ räumte ein Sprecher der DAK-Gesundheit ein, dass „in wenigen Einzelfällen“ wechselwillige Mitglieder vom Verbleib überzeugt werden sollten. In zwei Fällen zahlten Kundenbetreuer den Versicherten „ohne Rechtsgrund“ einen Bonus von 85 Euro. In vier weiteren Fällen sei eine Prämie für den Verbleib in Aussicht gestellt worden, die aber nach Angaben des Sprechers nicht ausgezahlt wurde.

Für die DAK-Gesundheit läuft es derzeit nicht rund: In den vergangenen Monaten hatten mehrere Medien von einem rasanten Mitgliederverlust bei Deutschlands drittgrößter gesetzlicher Krankenkasse berichtet. Seit Jahresbeginn erhebt die Kasse mit 1,5 Prozentpunkten den zweithöchsten Zusatzbeitrag. Dadurch habe die DAK-Gesundheit der Vereinten Dienstleistungsgewerkschaft ver.di zufolge mehr als 180.000 ihrer insgesamt sechs Millionen Mitglieder verloren. Mitte des Jahres wurde bekannt, dass die Kasse bundesweit 1600 von insgesamt 10.000 Vollzeitstellen abbaut. cas 